\title{
Telmisartan is More Effective than Taurine in Protecting High Fat Diet Induced Obesity in Rats from Hypertension, Some Metabolic, Oxidative Stress and Vascular Complications
}

Maha Mohamed El Batsh ${ }^{1}$ and Manal Mohamed El Batch ${ }^{2^{*}}$

${ }^{1}$ Department of Clinical Pharmacology, Faculty of Medicine, Menofiya University, Egypt

${ }^{2}$ Department of Medical Biochemistry, Faculty of Medicine, Tanta University, Egypt

\begin{abstract}
Obesity, insulin resistance, hypertension and fatty liver, are key risk factors for vascular complications. So, this study aimed to compare between telmisartan and taurine supplementation on systolic blood pressure (SBP) in addition to some metabolic disturbances and some vascular complications in an animal model for obesity.

Methods: Sixty male Wistar rats were randomly divided into six groups $(n=10)$ for 8 weeks three groups of them received standard diet with either vehicle or taurine $(3 \% \mathrm{w} / \mathrm{v}$ in drinking water) or telmisartan $(5 \mathrm{mg} / \mathrm{kg}$, oral) while the other three groups received high fat diet with either vehicle or taurine or telmisartan.

Results: The high fat diet group had greater body weight and higher SBP as compared to control rats. Increased plasma glucose, lipid profile (except HDL), insulin, insulin resistance, MDA, and ADMA but decreased HDL, PON-1 and DDAH were also observed. Telmisartan or taurine administration resulted in decreased SBP, plasma glucose lipid profile, insulin, insulin resistance, MDA, and ADMA but increased both plasma HDL level and PON-1activity, in addition to kidney DDAH enzyme activity with more significant effect of telmisartan than taurine.

Taken together, these results support the more beneficial effect of telmisartan than taurine in obese rats by improving SBP, in addition to ameliorating hyperglycemia , dyslipidemia,( metabolic disturbances) and decreasing plasma ADMA but increasing kidney DDAH enzyme activities (vascular complications) at least in part, by improving insulin sensitivity and decreasing oxidative stress, suggesting the possible use of telmisartan as a protective strategy against vascular complications related to obesity.
\end{abstract}

Keywords: ADMA; Oxidative stress; Obesity; Taurine; Telmisartan

\section{Introduction}

Obesity, an important component of metabolic syndrome (MS) which is characterized by the presence of multiple risk factors for the development of cardiovascular disease including hypertension, insulin resistance (IR), and dyslipidaemia, is a chronic low-grade inflammatory condition leading to adipocyte differentiation and growth in adipose tissues [1]. Accumulated adipose tissue is a metabolically active organ that secretes several substances called adipokines. These adipokines are mostly related, directly or indirectly to the link between adiposity and MS [2].

One of the recent adipokine that human adipocytes released is called asymmetric dimethylarginine (ADMA) which is a very wellknown risk factor of cardiovascular complications. ADMA is an endogenous inhibitor of all isoforms of nitric oxide synthase (NOS) [3], thus, ADMA can reduce nitric oxide (NO) synthesis, thus playing an important role in the reactive oxygen species (ROS) ROS/NO imbalance, that has been implicated in the pathogenesis of hypertension [4]. ROS induces ADMA accumulation by inhibiting dimethyl arginine dimethylaminohydrolase (DDAH), an enzyme responsible for the metabolism of ADMA. It is mainly located in the liver but it can also be found in other organs such as kidneys, brain, adrenal glands, pancreas or testes [5].

While obesity-induced vascular dysfunction appears to be reversible, at least in part, with weight control strategies, these have not proved sufficient to prevent the metabolic and cardiovascular complication of obesity on a large scale, therefore, elucidation of the pathophysiological mechanisms underlying vascular damage in obese patients is necessary to identify additional pharmacologic targets to prevent the cardiovascular complications of obesity [6].

To achieve optimal prevention of cardiovascular outcomes in obese patients, a multi-factorial approach is required, combining lifestyle modification, blood pressure lowering, and control of dyslipidemia [7]. For instance, several studies have shown that telmisartan, an angiotensin receptor blocker (ARB) and partial agonist of peroxisome proliferator-activated receptor-gamma (PPAR- $\gamma$ ), can reduce blood pressure as well as improve IR in obese subjects [8]. However, the mechanisms by which it acts are still unknown.

Taurine (2-aminoethylsulphonic acid) is a non-protein amino acid present in almost all animal tissues [9]. It exerts a diverse array of biological effects, including cardiovascular regulation, anti-oxidation, modulation of ion transport, membrane stabilization and modulation of neurotransmission [10]

So the aim of this work is to compare between the effects of

*Corresponding author: Maha Mohamed El Batsh, Department of Clinical Pharmacology, Faculty of Medicine, Menofiya University, Egypt, Tel: +201090012278; E-mail: mahaelbatsh@yahoo.com

Received June 03, 2014; Accepted July 02, 2014; Published July 06, 2014

Citation: EIBatsh MM, EIBatch MM (2014) Telmisartan is More Effective than Taurine in Protecting High Fat Diet Induced Obesity in Rats from Hypertension Some Metabolic, Oxidative Stress and Vascular Complications. Cardiol Pharmacol 3: 119. doi:10.4172/2329-6607.1000119

Copyright: (C) 2014 ElBatsh MM et al. This is an open-access article distributed under the terms of the Creative Commons Attribution License, which permits unrestricted use, distribution, and reproduction in any medium, provided the origina author and source are credited. 
telmisartan and taurine on not only blood pressure, body weight, some metabolic parameters (plasma glucose, insulin, IR, lipid profile), but also on some oxidative stress parameters, plasma ADMA and kidney DDAH enzyme activity on high fat diet induced obesity in rat.

Such a finding would suggest that either telmisartan or taurine supplementation might provide health benefits in obese patients currently receiving treatment, and might even help to prevent complications of obesity.

\section{Materials and Method}

\section{Chemicals}

Biochemicals, substrates and taurine were purchased from SigmaAldrich Chemicals (St. Louis, MO), telmisartan (Micardis) purchased from Boehringer Ingelheim Inc.

Taurine (3\% w/v) was administered to rats (Group II and Group V). Telmisartan tablets were crushed and dissolved in water then administered in drinking water to rats (Group III and Group VI). The concentration of telmisartan in drinking water was adjusted so that each rat ingested approximately $5 \mathrm{mg} / \mathrm{kg} /$ day. Both taurine and telmisartan were freshly prepared and chosen according to $[11,12]$ respectively.

\section{Animals}

Sixty male Wistar rats were used for the present study. The animals were acclimatized for two weeks before dietary manipulation. Four rats were housed per cage with constant 12 hours light and dark cycle and controlled temperature $\left(23 \pm 2^{\circ} \mathrm{C}\right)$.

\section{Experimental design}

Animals weighed 170-230 grams at the beginning of the study. They were randomly assigned into six groups of ten each $(n=10)$, as follow:

i. Control group (G-I): received standard diet and did not receive any medication

ii. Taurine group (G-II): received taurine $(3 \% \mathrm{w} / \mathrm{v})$ in drinking water and standard diet.

iii. Telmisartan group (G-III): received telmisartan $(5 \mathrm{mg} / \mathrm{kg})$ in drinking water and standard diet

iv. High fat diet group (G-IV): received high fat diet (HFD).

v. High fat diet+taurine group $(\mathrm{G}-\mathrm{V})$ : received taurine $(3 \% \mathrm{w} / \mathrm{v})$ in drinking water, and HFD.

vi. High fat diet+telmisartan group (G-VI): received telmisartan (5 $\mathrm{mg} / \mathrm{kg}$ ) in drinking water, and HFD.

The animals were maintained in their respective groups for 8 weeks.

\section{Diet}

Food and water were supplied ad libitum. All rats were fed a standard rat chow before beginning the study and continued to consume standard rat chow composed of $(23.3 \%$ protein, $66.5 \%$ carbohydrate $10.2 \%$ fat, and $13.4 \mathrm{~kJ} / \mathrm{g}$ ) [36] for control group (GI), taurine group (G-II) and telmisartan group (G-III). Following acclimatization, both G-IV, G-V and G-VI were given high-fat formulated diet $56 \%$ high-fat food $(7.0 \%$ protein, $37 \%$ carbohydrate, and $56 \%$ fat, $21.8 \mathrm{~kJ} / \mathrm{g}$ ) and water as suggested by [13]. Both standard rat chow and high-fat diet contained a standard mineral and vitamin mixture. Water and the dietary regimes were given ad libitum for 8 weeks. The body weight, food, and water intakes were monitored twice weekly throughout the study period ( 8 weeks). Dead rats were excluded from the study.

\section{Measurement of blood pressure}

It was measured by indirect method in which systolic blood pressure (SBP) was measured in $\mathrm{mmHg}$, weekly, using the noninvasive tail plethysmography method (plethysmograph LE5001; Panlab;, Barcelona, Spain). After preconditioning in the containment chamber, the animals were preheated to $35 \pm 2{ }^{\circ} \mathrm{C}$ for 5 minutes and at least five measurements of each rat were taken at 5 minutes intervals and the mean value was calculated [14].

All animals received appropriate care in compliance with the Public Health Service Policy on Use of Laboratory Animals published by the National Institutes of Health and was approved by the Ethical Committee of the Faculty of Medicine, Menoufia University, Egypt.

\section{Sample preparations of plasma and kidney tissue}

After 8 weeks of diet supplementation and treatment, rats were fasted for 12 hours and then sacrificed, then:

a. Blood samples were collected on heparin as an anticoagulant. Plasma samples were centrifuged at $4,000 \times \mathrm{g}$ for $30 \mathrm{~min}$ and then stored at $-70^{\circ} \mathrm{C}$ for further analysis.

b. The kidneys, were removed and rinsed with ice cold $0.9 \%$ saline solution to remove blood debris, then they were dried with filter paper, and their weights were measured, and stored in aluminum foil, wrapped at $-70^{\circ} \mathrm{C}$ until used for homogenization.

Specimens of kidney tissues were homogenized in 10 volumes of phosphate buffer saline (PBS) $0.1 \mathrm{M}, \mathrm{pH} 6.5$ using a potter-Elvenhjem tissue homogenizer (30 up and down strokes). The crude homogenate was centrifuged at $7,700 \mathrm{Xg}$ for 30 minutes at $4^{\circ} \mathrm{C}$, and the resultant supernatant was collected and stored at $-70^{\circ} \mathrm{C}$ until the analysis of DDAH enzyme activity and protein content according to Tain and Baylis [15] and Bradford [16] respectively.

\section{Laboratory analysis of all samples includes}

Fasting plasma samples were used for the measurement of:

1. Glucose level by the glucose oxidase method using a commercial kit (Elitech Diagnostics Company, France) according to the manufacturer's instructions.

2. Total cholesterol (TC) and triacylglycerol (TAG) levels using the appropriate kits (Boehringer Mannheim, Germany) according to the manufacturer's instructions. High density lipoprotein-cholesterol (HDL-C), was assessed after precipitation by phosphotungestic acid and magnesium chloride. Plasma LDL-C values were calculated using the Friedewald formula ([17] as follows: LDL-C=TC-(HDL$\mathrm{C}+\mathrm{TG} / 5)$. The tests utilized the principle of enzymatic colorimetric assay to read the sample.

3. Insulin, by Enzyme-Linked Immunosorbent Assay (ELISA) using commercial kit supplied by Sigma Scientific Service Company according to [18].

4. Homoeostasis model (HOMA-IR), assessment was used to estimate 
Citation: Batsh MMEI, Batch MMEI (2014) Telmisartan is More Effective than Taurine in Protecting High Fat Diet Induced Obesity in Rats from Hypertension, Some Metabolic, Oxidative Stress and Vascular Complications. Cardiol Pharmacol 3: 119. doi:10.4172/2329-6607.1000119

Page 3 of 6

insulin resistance, using the following equation: HOMA-IR=[Fasting plasma insulin $((\mu \mathrm{IU} / \mathrm{mL})$. Fasting plasma glucose $(\mathrm{mg} / \mathrm{dL}) / 405)]$ according to [19].

5. Malondialdehyde (MDA) according to Halliwell and Chirico [20], which depends on MDA reaction with thiobarbituric acid producing thiobarbituric acid reactive substance, a pink chromogen, which can be measured spectrophotometrically at $532 \mathrm{~nm}$. MDA was expressed as $\mathrm{nmol} / \mathrm{ml}$ and was calculated using $1.65 \mathrm{X} 105 \mathrm{M}-1 . \mathrm{cm}-1$ as molar absorption coefficient.

6. Paraoxonase-1(PON-1)activityusingsyntheticdiethyl-p-nitrophenyl phosphate (paraoxon, o,o-diethyl-pnitrophenylphosphate, Sigma Chemical Co., London, UK) and $\mathrm{CaCl} 2(1 \mathrm{mM}$ in $0.1 \mathrm{M}$ Tris buffer, $\mathrm{pH}$ 7.4) as moderator. The activity toward paraoxon was determined by measuring the initial rate of substrate hydrolysis to p-nitrophenol. In brief, reaction (final volume $1100 \mu \mathrm{l}$ ) for the hydrolysis of paraoxon contained $200 \mu \mathrm{l}$ of $0.1 \mathrm{M}$ Tris buffer $(\mathrm{pH}$ 7.4), $2.0 \mathrm{mM} \mathrm{CaCl} 2$ and $800 \mu \mathrm{l}$ of $1 \mathrm{mM}$ paraoxon solution to which $100 \mu \mathrm{l}$ undiluted plasma was added to start the reaction. Increase in absorbance at $405 \mathrm{~nm}$ was monitored for 3-5 minutes. The blank sample containing incubation mixture without plasma was run in parallel to correct for spontaneous substrate breakdown. The enzyme activity was calculated from molar extinction coefficient at 405 of p-nitrophenol (16000l mol-1. cm-1) and was expressed in $\mu \mathrm{mol} \cdot \mathrm{min}-1 \cdot \mathrm{ml}-1[21]$.

7. ADMA using an ELISA Kit (DLD Diagnostika, Germany) supplied by New Test Scientific Service Company according to [22].

\section{Kidney homogenate of rats}

Was used for estimation of DDAH by a colorimetric assay measuring the rate of the conversion of ADMA to L-citrulline, according to Tain and Baylis [15]. Briefly, the tissue homogenates were incubated with urease for $15 \mathrm{~min}$, and then $100 \mu \mathrm{L}(2 \mathrm{mg})$ of homogenate was incubated with $4 \mathrm{mmol} / \mathrm{l}$ ADMA, $0.1 \mathrm{~mol} / \mathrm{l}$ sodium phosphate buffer ( $\mathrm{pH}$ 6.5) in a total volume of $0.5 \mathrm{ml}$ for $3 \mathrm{~h}$ at $37^{\circ} \mathrm{C}$. The reaction was stopped by the addition of an equal volume of $10 \%$ trichloroacetic acid, and the supernatant was boiled with diacetylmonoxime $(0.8 \%$ $\mathrm{wt} / \mathrm{vol}$ in $5 \%$ acetic acid) and antipyrine (0.5\% wt/vol in 50\% sulfuric acid). The amounts of L-citrulline formed were determined with the spectrophotometric analysis at $466 \mathrm{~nm}$ and it was represented as $\mu \mathrm{m}$ L-citrulline formation $/ \mathrm{g}$ protein $/ \mathrm{min}$ at $37^{\circ} \mathrm{C}$. Protein determination was carried out by the method of [39].

\section{Statistical analysis}

Data were expressed as mean \pm SD. One-way ANOVA followed by a Tukey-Kramer post-hoc test was used for statistical comparison among the various treatment groups. Pearson correlation analysis was used to examine the relationship between ADMA, DDAH and other studied parameters. Tests were performed using Graph Pad Instat $\mathrm{P}<0.05$ was considered statistically significant.

\section{Results}

Comparison between the effects of taurine and telmisartan on body weight, SBP and metabolic parameters in high fat diet induced obesity in rat (Tables 1 and 2).

Body weight, SBP, fasting plasma glucose, lipid profile concentrations and insulin did not differ significantly between the studied groups at the beginning of the experiments (data not shown). Upon administration of high fat diet for 8 weeks, there was a significant increase in body weight, SBP, plasma glucose, TC, TAG, LDL-C,

\begin{tabular}{|c|c|c|c|c|c|c|c|}
\hline Variables & G-I $(n=10)$ & G-II $(n=10)$ & G-III $(n=10)$ & G-IV (n=10) & G-V $(n=10)$ & G-VI $(n=10)$ & $\mathbf{F}$ \\
\hline Final body weight in gm & $332.8 \pm 16.2$ & $335.2 \pm 18.4$ & $330 \pm 22$ & $366 \pm 19^{*}$ & $358 \pm 12^{*}$ & $356 \pm 13^{*}$ & 8.12 \\
\hline $\mathrm{SBP}(\mathrm{mmHg})$ & $120 \pm 11$ & $125 \pm 10$ & $118 \pm 12$ & $155 \pm 12^{*}$ & $137 \pm 8^{* \#}$ & $123 \pm 9^{\#-}$ & 18.2 \\
\hline
\end{tabular}

${ }^{*} \mathrm{p}<0.001$ vs. Group I; \# P<0.001 vs. Group IV; P<0.001 vs. Group V

G-I is control group, G-II is control group taken taurine, G-III is control group taken telmisartan G-IV, is high fat diet obese group, G-V is high fat diet obese group taken taurine, G-VI is high fat diet obese group taken telmisartan.

Table 1: Comparison between the effects of telmisartan and taurine on final body weight and SBP, in high fat diet induced obesity in rats.

\begin{tabular}{|c|c|c|c|c|c|c|c|}
\hline Variables & G-I $(n=10)$ & G-II $(n=10)$ & G-III $(n=10)$ & G-IV $(n=10)$ & G-V $(n=10)$ & G-VI $(n=10)$ & $\mathbf{F}$ \\
\hline $\mathrm{TC}(\mathrm{mg} / \mathrm{dl})$ & $95.8 \pm 3.9$ & $93.2 \pm 2.9$ & $90.5 \pm 3.4$ & $120.7 \pm 6.3^{*}$ & $114.5 \pm 4.9^{* \#}$ & $110 \pm 5.5^{\#^{*}}$ & 73.5 \\
\hline TAG (mg/dl) & $93.7 \pm 3.7$ & $90.6 \pm 4.1$ & $88 \pm 5$ & $140.5 \pm 8.5^{*}$ & $130 \pm 10^{* \#}$ & $127 \pm 9^{\#^{*}}$ & 106.1 \\
\hline LDL-C (mg/dl) & $29.5 \pm 2.2$ & $28.8 \pm 2.6$ & $27.5 \pm 3.1$ & $53.2 \pm 3.1^{\star}$ & $46.2 \pm 3.5^{\star \#}$ & $40.8 \pm 4.2^{* \# \sim}$ & 113.2 \\
\hline HDL-C (mg/dl) & $39 \pm 2$ & $40 \pm 2$ & $41 \pm 3$ & $29 \pm 3^{*}$ & $35 \pm 3^{* \#}$ & $37 \pm 4^{\#}$ & 22.8 \\
\hline FPG (mg/dl) & $101.5 \pm 7.8$ & $98.5 \pm 6.9$ & $99.5 \pm 6.5$ & $164.2 \pm 10.7^{\star}$ & $140.5 \pm 9.9^{* \#}$ & $138.7 \pm 8.9^{\star \#}$ & 104.7 \\
\hline Plasma insulin $(\mu \mathrm{lU} / \mathrm{ml})$ & $17.9 \pm 5.4$ & $16.2 \pm 4.8$ & $15.9 \pm 4.5$ & $36.4 \pm 6.3^{*}$ & $26.1 \pm 5.9^{* \#}$ & $19.2 \pm 3.9^{\#-}$ & 23.7 \\
\hline HOMA-IR & $5.3 \pm 1.3$ & $4.9 \pm 1.2$ & $3.9 \pm 1.6$ & $13.9 \pm 3.2^{*}$ & $8.4 \pm 1.7^{* \#}$ & $5.9 \pm 1.4^{\# \sim}$ & 39.1 \\
\hline $\mathrm{MDA}(\mu \mathrm{mol} / \mathrm{L})$ & $1.5 \pm 0.5$ & $1.3 \pm 0.2$ & $1.2 \pm 0.4$ & $3.5 \pm 0.1^{\star}$ & $2.1 \pm 0.5^{\star \#}$ & $1.8 \pm 0.3^{\#}$ & 54.3 \\
\hline $\mathrm{PON}-1(\mu \mathrm{mol} / \mathrm{min} / \mathrm{ml})$ & $38.9 \pm 9.5$ & $39.5 \pm 8.6$ & $40.2 \pm 8.2$ & $18.2 \pm 6.5^{\star}$ & $31.5 \pm 7.9^{\#}$ & $32.8 \pm 7.2^{\#}$ & 10.8 \\
\hline ADMA $(\mu \mathrm{mol} / \mathrm{L})$ & $1.03 \pm 0.29$ & $0.9 \pm 0.26$ & $0.8 \pm 0.28$ & $2.5 \pm 0.3^{*}$ & $2.1 \pm 0.25^{\star \#}$ & $1.7 \pm 0.2^{* \# \sim}$ & 70.2 \\
\hline $\mathrm{DDAH}(\mu \mathrm{M} / \mathrm{g}$ protein/min) & $0.5 \pm 0.04$ & $0.48 \pm 0.03$ & $0.51 \pm 0.02$ & $0.18 \pm 0.01^{*}$ & $0.24 \pm 0.01^{* \#}$ & $0.30 \pm 0.02^{* \# \sim}$ & 365.1 \\
\hline
\end{tabular}

* $p<0.001$ vs. Group I; \# P $<0.001$ vs. Group IV; $\sim P<0.001$ vs. Group V

G-I is control group, G-II is control group taken taurine, G-III is control group taken telmisartan G-IV, is high fat diet obese group , G-V is high fat diet obese group taken taurine, G-VI is high fat diet obese group taken telmisartan. Fasting plasma glucose (FPG), total cholesterol (TC), Triacylglycerol (TAG), High density lipoproteincholesterol (HDL-C) Low density lipoprotein-cholesterol (LDL-C), HOMA-IR homeostatic model assessment of insulin resistance, paraoxonase-1 (PON-1), asymmetric dimethylarginine (ADMA), Dimethyl arginine dimethylaminohydrolase (DDAH)

Table 2: Comparison between the effects of telmisartan and taurine on some metabolic, oxidative stress and vascular complications parameters in high fat diet induced obesity in rats. 
insulin and insulin resistance (HOMA-IR) but significant lower level of HDL-C in obese rats (group IV) as compared to control groups. With administration of either taurine or telmisartan, there was a significant decrease in all the studied parameters except for HDL-C (significant increase) as compared to obese rats with no significant difference in body weight (Table 1). These findings indirectly suggested that the reduced plasma glucose and lipid profile in either taurine or telmisartan group resulted from the physiological effects of either taurine or telmisartan itself, rather than from weight loss. Further-more, there was a significant difference between telmisartan and taurine treated groups in some parameters (SBP, LDL-C, plasma insulin, HOMA-IR) with more significant improvement in telmisartan rather than taurine treated group.

Comparison between the effects of taurine and telmisartan on oxidative stress and vascular complication parameters in high fat diet induced obesity in rat (Table 2).

There was a significant increase in plasma MDA, and ADMA but significant decrease in plasma PON-1, and kidney DDAH enzyme activities in obese group (group IV) as compared to control group. With administration of either taurine or telmisartan, there was a significant decrease in plasma MDA, and ADMA but significant increase in plasma PON-1, and kidney DDAH enzyme activities in either taurine or telmisartan treated group as compared to obese group, with more significant effect with telmisartan than taurine especially regarding ADMA and DDAH (Table 2).

Significant correlation between both ADMA and kidney DDAH enzyme activity and other studied parameter in obese rats (with or without taurine or telmisartan administration, $n=30$ ) was shown in Table 3.

\section{Discussion}

Obesity plays a central role in the IR syndrome the mechanisms linking obesity and increased cardiovascular risk profile is not well understood. Besides IR, it has been suggested that oxidative stress and ADMA may contributes to vascular damage. So, the present study was designed to be a trial to protect obese rats from some vascular complications by comparing the effects of telmisartan and taurine supplementation, on SBP, some metabolic disturbances and some vascular complications.

The initial characterization of the present study had shown that high fat diet induced-obesity in rats leads to hypertension. Activation of the

\begin{tabular}{|l|c|c|c|c|}
\hline \multirow{2}{*}{$\mathbf{n}=30$} & \multicolumn{2}{|c|}{ DDAH $(\boldsymbol{\mu M} / \mathbf{g}$ protein/min) } & \multicolumn{2}{c|}{ ADMA $(\boldsymbol{\mu m o l} / \mathbf{L})$} \\
\cline { 2 - 5 } & $\mathbf{r}$ & $\mathbf{P}$ value & $\mathbf{r}$ & $\mathbf{P}$ value \\
\hline Weight $(\mathrm{grams})$ & -0.52 & $<0.05$ & 0.50 & $<0.01$ \\
\hline SBP $(\mathrm{mmHg})$ & -0.59 & $<0.001$ & 0.62 & $<0.001$ \\
\hline TAG $(\mathrm{mg} / \mathrm{dl})$ & -0.56 & $<0.01$ & 0.56 & $<0.01$ \\
\hline HDL $(\mathrm{mg} / \mathrm{dl})$ & 0.55 & $<0.01$ & -0.53 & $<0.01$ \\
\hline LDL $(\mathrm{mg} / \mathrm{dl})$ & -0.62 & $<0.001$ & 0.54 & $<0.01$ \\
\hline TC $(\mathrm{mg} / \mathrm{dl})$ & -0.50 & $<0.01$ & 0.55 & $<0.01$ \\
\hline Glucose $(\mathrm{mg} / \mathrm{dl})$ & -0.45 & $>0.05$ & 0.55 & $<0.01$ \\
\hline Plasma insulin $(\mu \mathrm{lU} / \mathrm{ml})$ & -0.55 & $<0.01$ & 0.59 & $<0.001$ \\
\hline HOMA-IR & -0.58 & $<0.001$ & 0.58 & $<0.001$ \\
\hline MDA $(\mu \mathrm{mol} / \mathrm{L})$ & -0.66 & $<0.001$ & 0.68 & $<0.001$ \\
\hline PON $(\mu \mathrm{mol} / \mathrm{min} / \mathrm{ml})$ & 0.70 & $<0.001$ & -0.68 & $<0.001$ \\
\hline ADMA $(\mu \mathrm{mol} / \mathrm{L})$ & -0.72 & $<0.001$ & ----- & ------ \\
\hline
\end{tabular}

Table 3: Correlation between ADMA, kidney DDAH enzyme activity and other studied parameter in obese rats (with or without taurine or telmisartan administration) $(n=30)$. sympathetic nervous system contributes to blood pressure elevation in obesity [23]. Furthermore, hypertension is considered as a major determinant of endothelial dysfunction and angiotensin II receptor 1 antagonists are shown to possess antihypertensive effect. Substantial evidences suggested that telmisartan is a partial PPAR $\gamma$ agonist and thus it may efficiently improve endothelial function [24]. In the current study, both taurine and telmisartan significantly decreased the SBP as compared to the group IV, with more significant improvement in telmisartan treated group. Xu and Liu [25] and Hu et al. [26] stated that, telmisartan enhanced NO release by activating the PI3K/Akt system, AMPK phosphorylation and eNOS expression, which attenuated the blood pressure and oxidative stress in spontaneously hypertensive rats. On the other hand, taurine may decrease blood pressure by diminishing intracellular production of superoxide anions, which oxidizes $\mathrm{NO}$ after it is synthesized.

Another important finding in the present study is that obesity in rats leads to dyslipidemia which was corrected with administration of either taurine or telmisartan. The increase in TC, LDL-C, and TAG as well as decrease in HDL-C concentrations may be due to reduced lipoprotein lipase activity secondary to reduced plasma insulin levels [27]. Although, PPAR $\gamma$ regulates lipid metabolism and telmisartan exerts partial agonistic activity on PPAR $\gamma$, the control of dyslipidemia might be due to a direct angiotensin II type 1(AT-1) receptor inhibition [7]. The beneficial effects of taurine upon lipid metabolism in the present study can be explained by either increases of cholesterol $7 a$-hydroxylase activity in the liver, thus improving the conversion of cholesterol to bile acid formation [28] or by suppression in TAG secretion by the liver, inhibition of LDL oxidation and reduction of oxidative stress [29].

It is well known that a high-fat diet leads not only to increased body fat accumulation, but also to IR [27]. Wellen and Hotamisligil [30] have linked the relation between obesity and IR in two different ways. First, ectopic lipid accumulation is a potential mechanism for this relationship. Second, a systemic chronic inflammatory response in obesity, characterized by altered cytokine production and activation of inflammatory signaling pathways, is another mechanism. Furthermore, Xiao et al. [31] stated that lipid-induced protein kinase $\mathrm{C}(\mathrm{PKC}-\delta)$ translocation induced oxidative stress, which in turn activated I $\mathrm{B}$ kinase $\beta$ (IKK $\beta$ )/JNK1 and increased serine phosphorylation of insulin receptor substrates 1 and 2, resulting in IR. In the current study, both telmisartan and taurine supplementation are able to improve the hyper insulinemia and to guard against the increase in HOMA. Telmisartan may induce its beneficial metabolic effects by directly inhibiting the AT1 receptor. It has been established that AT1 receptor stimulation by angiotensin II contributes to IR and its associated deleterious metabolic profile [11]. The renin-angiotensin system (RAS) blockade's beneficial effect on IR has been explained partially by haemodynamic effects, with improved delivery of insulin and glucose to peripheral tissues, as well as by direct effects on glucose transport and insulin signaling pathways. Therefore, normalization of blood pressure by telmisartan in high fat diet induced obesity in rats could contribute to improved insulin action and a better metabolic profile, but the use of other antihypertensive drugs, such as calcium channel blockers [32], has not had a metabolic impact, suggesting that telmisartan improved IR via blood pressure independent mechanisms. Regarding taurine it performed its effect through prevention of oxidative stress induced by PKC activation and subsequent events [33]. Consistent with this, administration of taurine to obese rats had a significant effect on both the lipid peroxidation product MDA and PON-1 enzyme activity. In 
the present experiments, the higher MDA but lower PON-1enzyme activity in obese rats may be due to one of the following; increased production of free radicals associated obesity, presence of some inhibitors against the enzyme activity of PON-1 in circulating blood of obese patients such as glycosylated proteins or disturbance of the interaction of PON-1 with HDL affecting its activity. Such inactivation increased the risk of cardiovascular diseases by inability of destroying proinflammatory molecules involved in the initiation and progression of atherosclerotic lesions as PON-1 has antioxidant properties which prevent the oxidation of LDL that play a great role in atherosclerosis [34]. Therefore, there is increasing evidence linking the low PON1 activity to an increased likelihood of cardiovascular diseases, so, enhancing PON-1 activity could be an important target for future pharmacological agents aimed at decreasing cardiovascular risk. In the present study, both telmisartan and taurine supplementation decreased MDA that could be explained on the bases of the direct anti-oxidant effects of either telmisartan or, taurine through scavenging hydroxyl radicals, hydrogen peroxide, singlet oxygen, superoxide radicals, and peroxyl radicals thereby preventing the free radicals-mediated lipid peroxidation $[35,36]$. Furthermore, because lipids are substrates for lipid peroxidation, the observed decrement in serum TAG levels in the current study could be another contributing factor to the reduced MDA levels. Because lipid peroxidation products have been reported to inhibit PON activity, the reduced plasma MDA levels, which reflected an improvement in the oxidative-anti-oxidative balance, may be a reason for or a contributing factor to the increased plasma PON-1 activities present in this current study [37].

Further-more it has been established that IR is associated with a reduction in NO production and impaired endothelial function [38]. Also, there was a relationship between ADMA and IR as stated by Miyazaki et al. [39], who found that patients with the worst insulin tolerance had the highest plasma concentrations of ADMA. Of note, a recent study in rheumatoid arthritis patients by Dimitroulas et al. [40] has revealed a close relationship between circulating ADMA concentrations and IR and concluded that, HOMA, a strong indicator of IR, seems to be the main predictor of elevated ADMA levels in rheumatoid arthritis patients.

In the present study, plasma ADMA has been significantly increased but kidney DDAH enzyme activity has been significantly decreased in obese rats, that has been reversed by either telmisartan or taurine. Diep et al. [41] stated that metabolic disorders, lipid metabolism abnormalities, hypertension, cerebrovascular/cardiovascular disorders, and highfat diet can result in increased ADMA levels. Moreover, Korandji et al. [42], suggested that the elevated levels of ADMA observed in rat model of MS could in part be secondary to the early development of oxidative stress associated with the development of hypertension. ADMA has been considered not only as a biochemical marker of vascular dysfunction, but also a novel target for treatment. In the concurrent study, telmisartan significantly decreased the blood levels of ADMA. Therefore, telmisartan can be expected to improve vascular endothelial function, at least in part, via a decrease in ADMA levels. The mechanism by which telmisartan decreased the ADMA levels is not well understood, but telmisartan-related decreases in blood pressure, plasma glucose, and lipids may be involved in this mechanism [43]. Also, telmisartan activates PPAR- $\gamma$ which may cause improvement of IR and thus inhibiting atherosclerosis. Based on this background, ADMA, as an intrinsic substance, may be clinically simple and useful for evaluating vascular endothelial function. ADMA is metabolized by DDAH. In the present study, administration of telmisartan resulted in significant ameliorationin the high fat diet induced reduction of
DDAH levels in rat kidneys. This suggests that elevated plasma NO levels by telmisartan treatment in the present animal model resulted, at least partially, from the improvement of plasma ADMA up-regulation and of kidney DDAH down-regulation.

Finally, regarding taurine effect in ADMA and DDAH, it decreases TC and LDL concentrations which would seem to preclude important role of these lipids in ADMA levels as mentioned by Boger et al. [44], who demonstrated that LDL-C up-regulated the synthesis of ADMA in human endothelial cells through the induction of protein arginine methyl transferase (PRMT), an enzyme associated with ADMA production. Furthermore the activity of DDAH seems to be related to oxidative stress. A wide range of pathologic stimuli induce endothelial oxidative stress and consequently reduce DDAH activity in vitro and in vivo. The adverse effect of these stimuli can be reversed in vitro by antioxidants like taurine, which preserve the activity of DDAH [45].

Also, our data demonstrate a correlation between plasma ADMA and fasting insulin levels (Table 3), suggesting that either telmisartan or taurine action may be directly on DDAH or indirect action through the change in insulin sensitivity.

Accumulating evidence has demonstrated a close association between elevated ADMA levels and cardiovascular risk factors [34]. Therefore, the decrease in ADMA levels by treatment with either telmisartan or taurine (with more significant effect of telmisartan) may contribute to cardiovascular risk reduction, so that telmisartan may constitute a novel therapeutic strategy in the field of cardiovascular disease.

\section{Conclusion}

The main finding in the present study is that the protective effect of either telmisartan or taurine in high fat diet induced obesity in rats from vascular complications may be related to reduction of ADMA concentration via increasing DDAH activity, decreasing MDA, increasing PON-1 and improvement of insulin sensitivity, with more effect in telmisartan than taurine treated group as indicated by the significant differences between telmisartan and taurine treated group in some parameters (SBP, plasma insulin, HOMA-IR, ADMA and DDAH). Also, telmisartan had more significant effect than taurine in blocking the development of hypertension as one of the associated vascular complications in high fat diet induced obesity in rats by either reduction of plasma ADMA or attenuation of oxidative stress. This suggested that telmisartan changes disturbed NO/ROS balance toward augmentation of NO leading to lowering of SBP. So, the results of the present study suggested that telmisartan may be superior to taurine in providing important health benefits in obese patients.

Thus either telmisartan or taurine may become interesting therapeutic agents for prevention of cardiovascular complications of obesity as well as for preventative therapy in other forms of cardiovascular disease. Further studies will elucidate the molecular mechanism by which telmisartan or taurine reversed the deleterious metabolic affects in this model.

\section{References}

1. Johnson AR, Milner JJ, Makowski L (2012) The inflammation highway: metabolism accelerates inflammatory traffic in obesity. Immunol Rev 249: 218-238.

2. Antuna-Puente B, Feve B, Fellahi S, Bastard JP (2008) Adipokines: the missing link between insulin resistance and obesity. Diabetes Metab 34: 2-11.

3. Bouras G, Deftereos S, Tousoulis D, Giannopoulos G, Chatzis G, et al 
Citation: Batsh MMEI, Batch MMEI (2014) Telmisartan is More Effective than Taurine in Protecting High Fat Diet Induced Obesity in Rats from Hypertension, Some Metabolic, Oxidative Stress and Vascular Complications. Cardiol Pharmacol 3: 119. doi:10.4172/2329-6607.1000119

(2013) Asymmetric Dimethylarginine (ADMA): a promising biomarker for cardiovascular disease? Curr Top Med Chem 13: 180-200.

4. Fan NC, Tsai CM, Hsu CN, Huang LT, Tain YL4 (2013) N-acetylcysteine prevents hypertension via regulation of the ADMA-DDAH pathway in young spontaneously hypertensive rats. Biomed Res Int: 696317.

5. Trocha M, Szuba A, Merwid-Lad A, SozaÅ,ski T (2010) Effect of selected drugs on plasma asymmetric dimethylarginine (ADMA) levels. Pharmazie 65: 562-571.

6. Campia U, Tesauro M, Cardillo C (2012) Human obesity and endotheliumdependent responsiveness. Br J Pharmacol 165: 561-573.

7. Kamari Y, Harari A, Shaish A, Peleg E, Sharabi Y, et al. (2008) Effect of telmisartan, angiotensin II receptor antagonist, on metabolic profile in fructoseinduced hypertensive, hyperinsulinemic, hyperlipidemic rats. Hypertens Res 31: $135-140$.

8. Komiya $\mathrm{N}$, Hirose $\mathrm{H}$, Kawabe $\mathrm{H}$, Itoh $\mathrm{H}$, Saito I (2009) Effects of telmisartan therapy on metabolic profiles and serum high molecular weight (HMW)adiponectin level in Japanese male hypertensive subjects with abdominal obesity. J Atheroscler Thromb 16: 137-142.

9. Kim SJ, Gupta RC, Lee HW (2007) Taurine-diabetes interaction: from involvement to protection. Curr Diabetes Rev 3: 165-175.

10. Abebe W, Mozaffari MS (2011) Role of taurine in the vasculature: an overview of experimental and human studies. Am J Cardiovasc Dis 1: 293-311.

11. Kumar V, Bhandari U, Tripathi CD, Khanna G (2013) Anti-obesity effect of Gymnema sylvestre extract on high fat diet-induced obesity in Wistar rats. Drug Res (Stuttg) 63: 625-632.

12. Kobayashi N, Ohno T, Yoshida K, Fukushima H, Mamada Y, et al. (2008) Cardioprotective mechanism of telmisartan via PPAR-gamma-eNOS pathway in dahl salt-sensitive hypertensive rats. Am J Hypertens 21: 576-581.

13. Xu L, Liu Y (2013) Administration of telmisartan reduced systolic blood pressure and oxidative stress probably through the activation of PI3K/Akt/eNOS pathway and NO release in spontaneously hypertensive rats. Physiol Res 62: 351-359.

14. Hu J, Xu X, Yang J, Wu G, Sun C, et al. (2009) Antihypertensive effect of taurine in rat. Adv Exp Med Biol 643: 75-84.

15. Zaman MQ, Leray V, Le Bloc'h J, Thorin C, Ouguerram K, et al. (2011) Lipid profile and insulin sensitivity in rats fed with high-fat or high-fructose diets. $\mathrm{Br}$ Nutr 106 Suppl 1: S206-210.

16. Choi MJ, Kim MJ, Chang KJ (2006) the effect of dietary taurine supplementation on plasma and liver lipid concentrations and mineral metabolism in rats fed alcohol.AdvExp Med Biol 583: 243-250.

17. Zhang M, Bi LF, Fang JH, Su XL, Da GL, et al. (2004) Beneficial effects of taurine on serum lipids in overweight or obese non-diabetic subjects. Amino Acids 26: 267-271

18. Wellen KE, Hotamisligil GS (2003) Obesity-induced inflammatory changes in adipose tissue. J Clin Invest 112: 1785-1788.

19. Xiao C, Giacca A, Lewis GF (2008) Oral taurine but not N-acetylcysteine ameliorates NEFA-induced impairment in insulin sensitivity and beta cell function in obese and overweight, non-diabetic men. Diabetologia 51: 139-146.

20. Goyal BR, Parmar K, Goyal RK, Mehta AA (2011) Beneficial role of telmisartan on cardiovascular complications associated with STZ-induced type 2 diabetes in rats. Pharmacol Rep 63: 956-966.

21. Oron-Herman M, Sela BA, Rosenthal T (2005) Risk reduction therapy for syndrome X: comparison of several treatments. Am J Hypertens 18: 372-378.

22. Imae M, Asano T, Murakami S (2014) Potential role of taurine in the prevention of diabetes and metabolic syndrome. Amino Acids 46: 81-88.

23. Koncsos P, Seres I, Harangi M, Illyés I, Józsa L, et al. (2010) Human paraoxonase- 1 activity in childhood obesity and its relation to leptin and adiponectin levels. Pediatr Res 67: 309-313.

24. Chaudagar KK, Mehta AA (2014) Effect of telmisartan on VEGF-induced and VEGF-independent angiogenic responsiveness of coronary endothelial cells in normal and streptozotocin (STZ)-induced diabetic rats. Clin Exp Hypertens.

25. Oliveira MW, Minotto JB, de Oliveira MR, Zanotto-Filho A, Behr GA, et al. (2010) Scavenging and antioxidant potential of physiological taurine concentrations against different reactive oxygen/nitrogen species. Pharmacol Rep 62: 185-93.

26. Aviram M, Rosenblat M, Billecke S, Erogul J, Sorenson R, et al. (1999) Human serum paraoxonase (PON 1) is inactivated by oxidized low density lipoprotein and preserved by antioxidants. Free Radic Biol Med 26: 892-904.

27. Siervo M Bluck LJ (2012) In vivo nitric oxide synthesis, insulin sensitivity, and asymmetric dimethylarginine in obese subjects without and with metabolic syndrome. Metabolism 61: 680-688.

28. Miyazaki H, Matsuoka H, Cooke JP, Usui M, Ueda S, et al. (1999) Endogenous nitric oxide synthase inhibitor: a novel marker of atherosclerosis. Circulation 99: 1141-1146.

29. Dimitroulas T, Sandoo A, Veldhuijzen van Zanten JJ, Smith JP, Hodson J, et al. (2013) Predictors of asymmetric dimethylarginine levels in patients with rheumatoid arthritis: the role of insulin resistance. Scand J Rheumatol 42: 176-181.

30. Diep QN, El Mabrouk M, Cohn JS, Endemann D, Amiri F, et al. (2002) Structure, endothelial function, cell growth, and inflammation in blood vessels of angiotensin II-infused rats: role of peroxisome proliferator-activated receptorgamma. Circulation 105: 2296-2302.

31. Korandji C, Zeller M, Guilland JC, Collin B, Lauzier B, et al. (2011) Time course of asymmetric dimethylarginine (ADMA) and oxidative stress in fructosehypertensive rats: a model related to metabolic syndrome. Atherosclerosis 214 310-315.

32. Scalera F, Martens-Lobenhoffer J, Bukowska A, Lendeckel U, Täger M, et al. (2008) Effect of telmisartan on nitric oxide--asymmetrical dimethylarginine system: role of angiotensin II type 1 receptor gamma and peroxisome proliferator activated receptor gamma signaling during endothelial aging. Hypertension 51: 696-703.

33. Böger RH, Bode-Böger SM, Szuba A, Tsao PS, Chan JR, et al. (1998) Asymmetric dimethylarginine (ADMA): a novel risk factor for endothelial dysfunction: its role in hypercholesterolemia. Circulation 98: 1842-1847.

34. Antoniades C, Shirodaria C, Leeson P, Antonopoulos A, Warrick N, et al (2009) Association of plasma asymmetrical dimethylarginine (ADMA) with elevated vascular superoxide production and endothelial nitric oxide synthase uncoupling: implications for endothelial function in human atherosclerosis. Eur Heart J 30: 1142-1150.

35. Du H, You JS, Zhao X, Park JY, Kim SH, et al. (2010) Antiobesity and hypolipidemic effects of lotus leaf hot water extract with taurine supplementation in rats fed a high fat diet. J Biomed Sci 17:42.

36. Machado H, Pinheiro HS, Terra MM, Guerra Mde O, de Paula RB, et al. (2012 [Dissociation of antihypertensive and metabolic response to losartan and spironolactone in experimental rats with metabolic sindrome]. J Bras Nefrol 34: $328-336$

37. Wang $Y$, Song $Y$, Suo M, Jin X, Tian G (2012) Telmisartan prevents high-fat diet-induced hypertension and decreases perirenal fat in rats. J Biomed Res 26: 219-225.

38. Tain YL, Baylis C (2007) Determination of dimethylarginine dimethylaminohydrolase activity in the kidney. Kidney Int 72: 886-889.

39. Bradford MM (1976) A rapid and sensitive method for the quantitation of microgram quantities of protein utilizing the principle of protein-dye binding Anal Biochem 72: 248-254.

40. Friedewald WT, Levy RI, Fredrickson DS (1972) Estimation of the concentration of low-density lipoprotein cholesterol in plasma, without use of the preparative ultracentrifuge. Clin Chem 18: 499-502.

41. Matveyenko AV, Veldhuis JD, Butler PC (2008) Measurement of pulsatile insulin secretion in the rat: direct sampling from the hepatic portal vein. Am J Physiol Endocrinol Metab 295: E569-574.

42. Duseja A, Thumburu KK, Das A, Dhiman RK, Chawla YK, et al. (2007) Insulin tolerance test is comparable to homeostasis model assessment for insulin resistance in patients with nonalcoholic fatty liver disease. Indian J Gastroenterol 26: 170-173

43. Halliwell B, Chirico S (1993) Lipid peroxidation: its mechanism, measurement and significance. Am J Clin Nutr 57: 715S-724S.

44. Bełtowski J, Wójcicka G, Marciniak A (2002) Species- and substrate-specific stimulation of human plasma paraoxonase (PON1) activity by high chloride concentration. Acta Biochim Pol 49: 927-936.

45. Schulze F, Wesemann R, Schwedhelm E, Sydow K, Albsmeier J, et al. (2004) Determination of asymmetric dimethylarginine (ADMA) using a novel ELISA assay. Clin Chem Lab Med 42: 1377-1383. 